\title{
Artificial Neural Networks for Single-Image Super-Resolution
}

\author{
Gagandeep Singh \\ Research scholar, \\ CSE Department \\ Chandigarh University, India
}

\author{
Gulshan Goyal \\ Assistant Professor, \\ CSE Department \\ Chandigarh University, India
}

\begin{abstract}
Image upscaling is an important field of digital image processing. It is often required to create higher resolution images from the lower resolution images at hand in computer graphics, media devices, satellite imagery etc. Upscaling is also referred to as 'single image super-resolution'. The process is a tradeoff between efficiency, time and the quality of output images obtained .In present paper, a feed forward neural network using supervised training for image upscaling is proposed. The performance of neural network is compared to bicubic interpolation method in terms of PSNR and MSE.
\end{abstract}

\section{General Terms}

Image Upscaling, Neural Networks.

\section{Keywords}

Upscaling, Neural Network, ANN, Super-Resolution, Interpolation, Resolution, Bicubic, Feed-Forward.

\section{INTRODUCTION}

Digital images are used in many fields like, medical imaging, satellite imagery, high-definition television broadcasts, printing etc. Some applications require use of high resolution images, however it's not always feasible to acquire images in high resolution. To obtain a higher resolution image from a lower resolution digital image is known as image upscaling or single image superresolution. Figure 1 shows an example of upscaling of image. Resolution has been frequently referred as an important aspect of an image. Images are being processed in order to obtain more enhanced resolution. One of the commonly used techniques for image resolution enhancement is Interpolation [1] Interpolation calculates the new pixel values for the new image with a higher spatial resolution. Interpolation does not add new details to the image though. There are several issues with the perceived quality of interpolated images: sharpness of edges, freedom from artifacts and reconstruction of high frequency details. [2]

There are various interpolation methods available for image resolution upscaling. There are linear or non-adaptive methods, like nearest neighbor interpolation, bilinear interpolation and bicubic interpolation. And then there are adaptive interpolation methods like edgedirected interpolation, decision adaptive interpolation. The adaptive methods take into account the presence of edges and curves in the subject image. The paper implements image upscaling via artificial neural networks using bicubic interpolation.
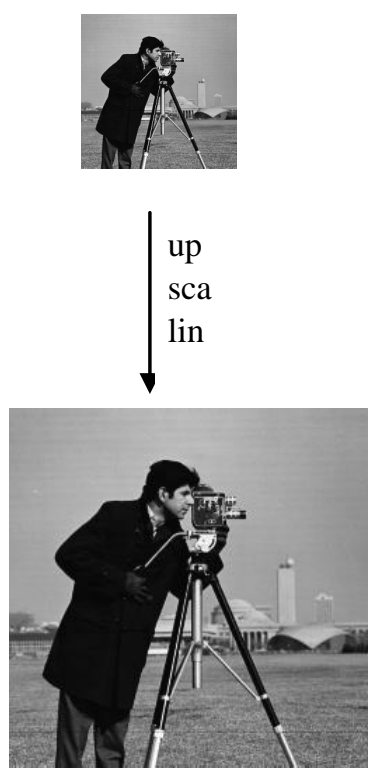

Figure 1: An example of image upscaling

\subsection{Need for image upscaling}

Scaling of images from lower resolution to a higher resolution is needed because of the following reasons:

1. It's easier to analyse and study higher resolution images.

2. Available sensors have limitation in respect to maximum resolution[3] So its needed to overcome some of the inherent resolution limitations of lowcost imaging sensors [4]

3. To produce images of high perceptual quality and produce visually appealing results

4. To keep the text and graphics as original as possible, while avoiding noise and artifacts in the image [5]

5. To preserve the nature and texture of image while enlarging it.

6. To allow for better utilization of the growing capability of High Resolution displays (e.g., HD LCDs). [5] 


\subsection{Applications of image upscaling}

Image upscaling is used in many areas, some of these are:

1. Printing-To scale and print images according to canvas size while maintaining picture quality.

2. Video playback-Televisions use real time up scaling algorithms to display simple definition content on high-resolution displays.

3. Mobile devices-To scale graphics and videos onto varying display sizes for viewing and playback.

4. Geosciences studies, astronomy, and geographical information systems. [6]

5. Computer Graphics-Computers perform screen image scaling which includes web pages, text, graphics, game scenes etc.[5]

6. Image processing packages-Image processing software use scaling for viewing and resizing the image. [7]

7. Satellite imaging-Nowadays, satellite images are used in many applications.
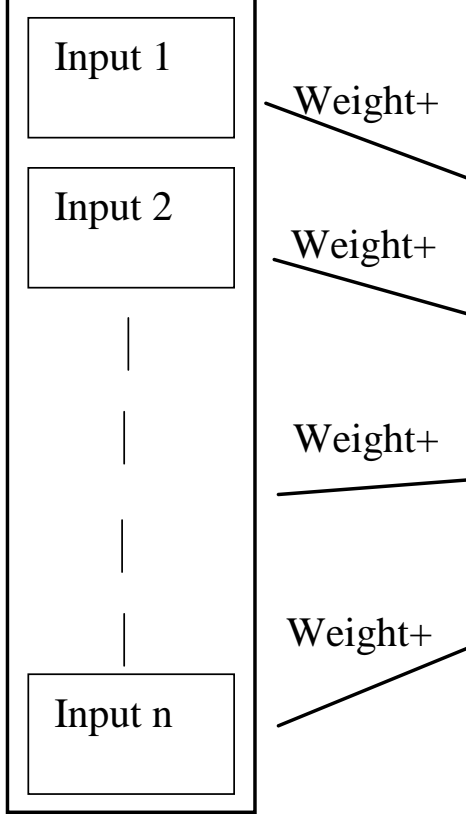

Weight +

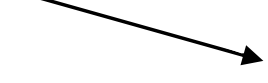

Weight+

Weight+

\section{IMAGE UPSCALING USING NEURAL NETWORKS}

An Artificial Neural Network (ANN) is an imitation of the way our biological neural network works. The nervous system contains numerous neurons that are linked to each other. These neurons play a key role in decision making and thought process. An artificial neural network aims to mimic the neural activity at a much smaller scale. An ANN consists of artificial neurons. Information processing takes place via these neurons.

Each neuron is connected to other neurons by means of directed communication links, each with an associated weight. The weights represent information being used by the net to solve a problem. [8]

Neural networks can be applied to a wide variety of problems, such as storing and recalling data or patterns, classifying patterns, performing general mappings from input patterns to output patterns, grouping similar patterns, or finding solutions to constrained optimization problems.[8]

\section{Input layers}

Figure 2: A simple Neural Network 


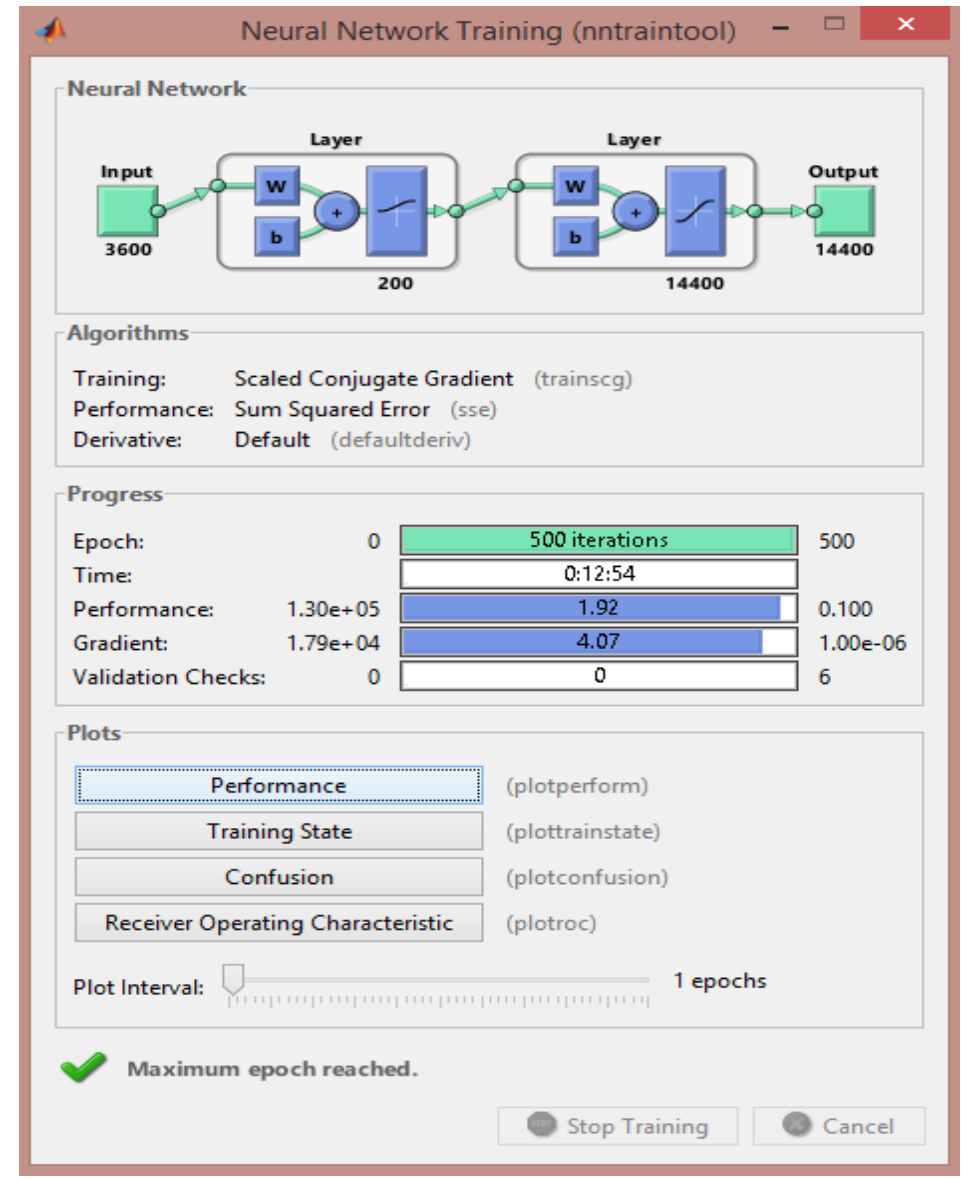

Figure 3: Training the neural network

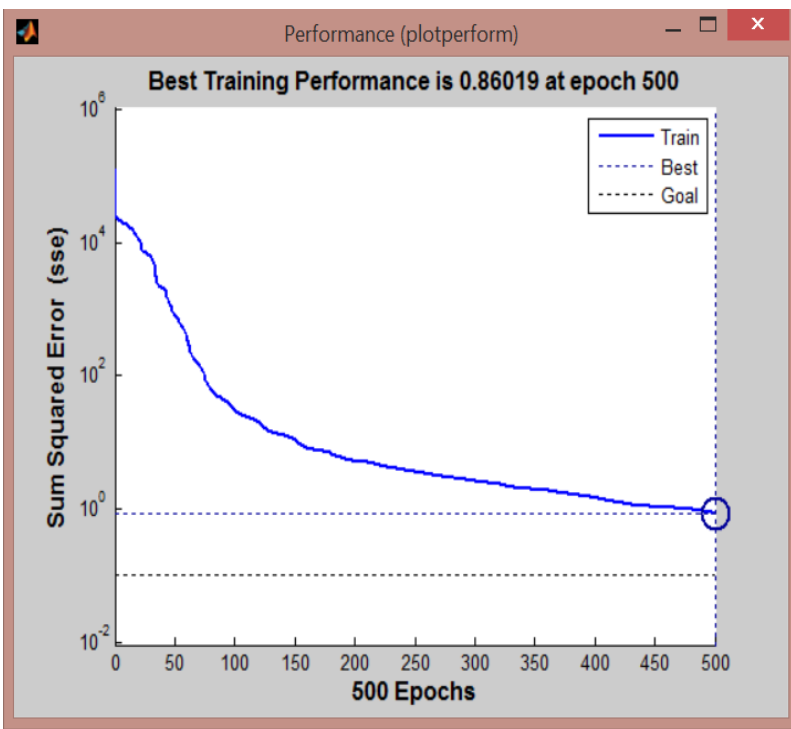

Figure 4: Performance graph

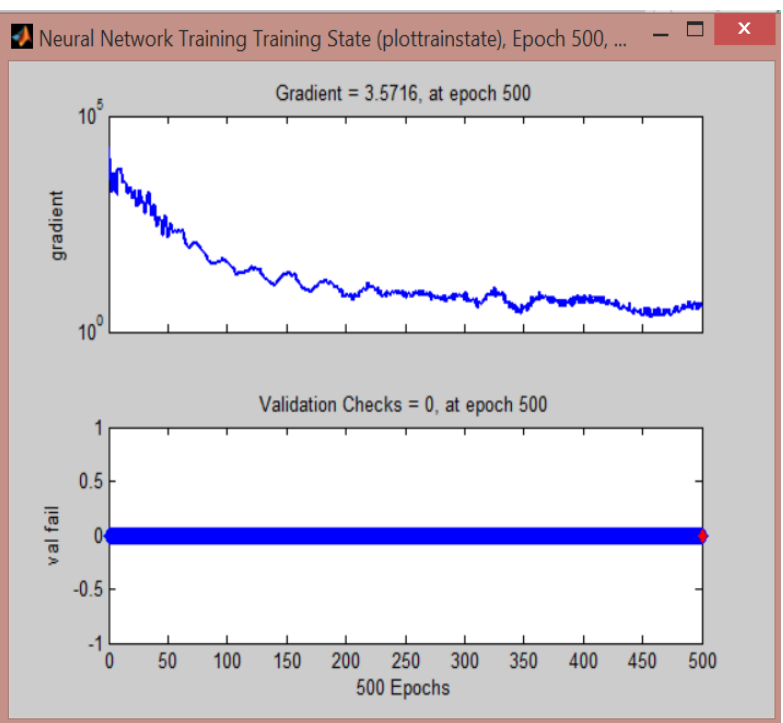

Figure 5: Gradient and validation check 
For implementing the neural network to upscale images, these network training parameters have been taken.
i. Number of neurons in the hidden layer $=200$
ii. Learning function=traingdx(adaptive learning function) and logsig (logarithmic sigmoid)
iii. Cost function $=\mathrm{SSE}$ (Sum Squared Error)
iv. Learning rate $=0.80$
v. Type of neural network used=newff (feed forward networks)
vi. Number of epochs $=500$
vii. Momentum constant $=0.95$
viii. Performance goal $=0.10$
ix. Input image $=60 * 60$ size image
x. Target image $=120 * 120$ upscaled image
xi. Type of learning used=supervised learning

The feed forward neural feed forward network with the above parameters was then applied on test images. The results produced by the trained neural network are displayed in table 1.

Table 1: Outputs of the Neural Network

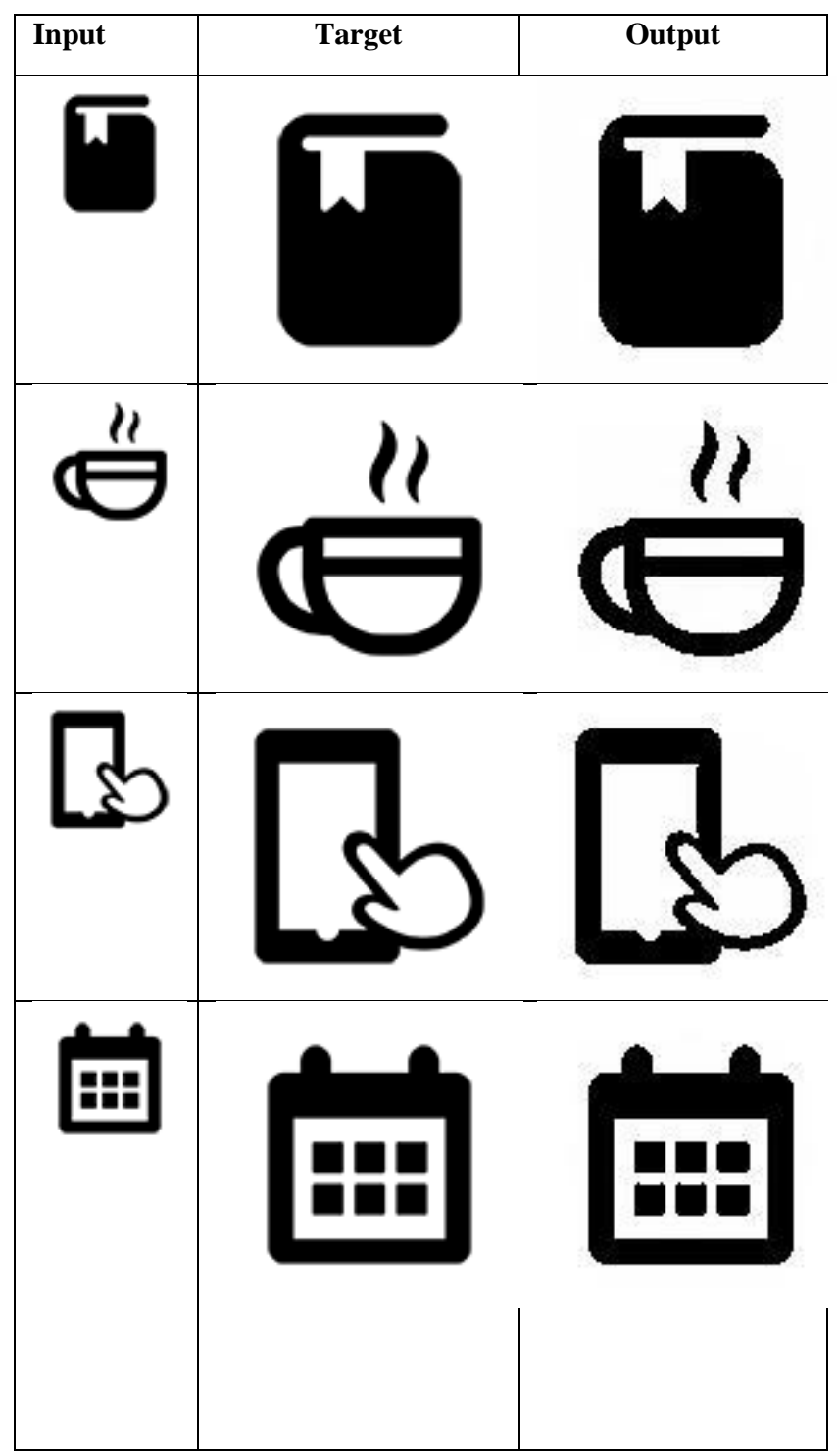

\begin{tabular}{|c|c|c|}
\hline Image & \multicolumn{2}{|c|}{ PSNR } \\
\hline & Bicubic & Bicubic with NN \\
\hline 1 & 52.0580 & 51.1506 \\
\hline 2 & 51.0695 & 51.2246 \\
\hline 3 & 50.9165 & 51.6892 \\
\hline 4 & 51.5507 & 52.4387 \\
\hline 5 & 52.8443 & 54.2087 \\
\hline 6 & 51.9893 & 51.3289 \\
\hline 7 & 50.8560 & 52.0899 \\
\hline 8 & 50.2909 & 50.5865 \\
\hline 9 & 49.3979 & 49.9825 \\
\hline 10 & 51.6589 & 52.6545 \\
\hline 11 & 49.6212 & 49.0763 \\
\hline 12 & 52.2196 & 51.9426 \\
\hline Average & 51.2060 & 51.531 \\
\hline
\end{tabular}

Table 2: Comparison of PSNR values

Table 3: Comparison of MSE values

\begin{tabular}{|c|c|c|}
\hline Image & \multicolumn{2}{|c|}{ MSE } \\
\hline & Bicubic & Bicubic with NN \\
\hline 1 & 0.0031 & 0.0039 \\
\hline 2 & 0.0040 & 0.0038 \\
\hline 3 & 0.0041 & 0.0034 \\
\hline 4 & 0.0035 & 0.0029 \\
\hline 5 & 0.0026 & 0.0019 \\
\hline 6 & 0.0032 & 0.0037 \\
\hline 7 & 0.0042 & 0.0031 \\
\hline 8 & 0.0047 & 0.0044 \\
\hline 9 & 0.0058 & 0.0051 \\
\hline 10 & 0.0034 & 0.0027 \\
\hline 11 & 0.0055 & 0.0063 \\
\hline 12 & 0.0030 & 0.0032 \\
\hline Average & 0.0039 & 0.0037 \\
\hline
\end{tabular}

The test bed was an AMD A8 Quad -core APU with 8 GB RAM running a 64-bit version of MATLAB. The dataset of 100 images was taken from website flaticon.com. Figure 3 shows the neural network, the number of layers, number of neurons and the functions used to train the network and the performance graphs generated

Table 1 shows a subjective analysis of the outputs of the trained neural network. Subjective analysis compares the image quality as seen by the observer. Table 2 presents the objective analysis of the methods. The evaluation parameters used for comparison are PSNR, MSE and RMSE.

The value of validation checks increases when the network is unable to learn over a span of iterations, Ideally the value should be zero.

A larger value of PSNR indicates better image output. MSE( Mean Square Error) and RMSE(Root Mean Square Error) should be minimum. A value less than one and closer to zero is desired for these parameters. Tables 2, 3, and 4 show a comparison of the values of these parameters after using the 
neural network. On an average, the application of the feed forward neural network has improved the signal to noise ratio and reduced the error values

Table 4: Comparison of RMSE values

\begin{tabular}{|c|c|c|}
\hline Image & \multicolumn{2}{|c|}{ RMSE } \\
\hline & Bicubic & $\begin{array}{c}\text { Bicubic with } \\
\text { NN }\end{array}$ \\
\hline 1 & 0.0564 & 0.0626 \\
\hline 2 & 0.0632 & 0.0621 \\
\hline 3 & 0.0643 & 0.0589 \\
\hline 4 & 0.0598 & 0.0540 \\
\hline 5 & 0.0515 & 0.0440 \\
\hline 6 & 0.0569 & 0.0614 \\
\hline 7 & 0.0648 & 0.0562 \\
\hline 8 & 0.0691 & 0.0668 \\
\hline 9 & 0.0766 & 0.0716 \\
\hline 10 & 0.0591 & 0.0527 \\
\hline 11 & 0.0747 & 0.0795 \\
\hline 12 & 0.0554 & 0.0572 \\
\hline Average & 0.0626 & 0.0605 \\
\hline
\end{tabular}

\section{CONCLUSION}

The trained neural network was able to reproduce higher resolution images from low resolution images. The PSNR values of outputs of neural network seem better than the ones obtained by just using bicubic interpolation. Similarly, the MSE and RMSE values for neural network are lesser, as desired. The implementation of the simple neural network has improved the two of these objective evaluation parameters. The subjective analysis also indicates good image output quality. For future work upscaling can be implemented using an back-propagation neural network over a larger dataset. The review was helpful in understanding the basics of neural networks and the possibility of implementing image upscaling via artificial neural networks.

\section{REFERENCES}

[1] Demirel, Hasan, and Gholamreza Anbarjafari. "Image resolution enhancement by using discrete and stationary wavelet decomposition." Image Processing, IEEE Transactions on 20.5 (2011): pp 1458-1460

[2] Su, Dan, and Philip Willis. "Image Interpolation by Pixel-Level Data-Dependent Triangulation." Computer Graphics Forum. Vol. 23. No. 2. Blackwell Publishing Ltd., 2004.

[3] Purkait, Pulak, and Bhabatosh Chanda. "Super resolution image reconstruction through Bregman iteration using morphologic regularization." Image Processing, IEEE Transactions on 21.9 (2012): 4029-4039.

[4] Yang, Jianchao, et al. "Coupled dictionary training for image super-resolution."Image Processing, IEEE Transactions on 21.8 (2012): 3467-3478.

[5] Zhang, Haichao, Yanning Zhang, and Thomas S. Huang. "Efficient sparse representation based image super resolution via dual dictionary learning."Multimedia and Expo (ICME), 2011 IEEE International Conference on IEEE, 2011.

[6] Schafer, Ronald W., and Russel M. Mersereau. "Demosaicking: color filter array interpolation." Signal Processing Magazine, IEEE 22.1 (2005).

[7] Han, Dianyuan. "Comparison of Commonly Used Image Interpolation Methods."ICCSEE, Hangzhou, China (2013)

[8] Fausett, Laurene. Fundamentals of neural networks: architectures, algorithms, and applications. Prentice-Hall, Inc., 1994. 